\title{
Evaluating the $\leq 10: 1$ wholegrain criterion in identifying nutrient quality and health implications of UK breads and breakfast cereals
}

\author{
Bahar Ghodsian ${ }^{1,2}$ and Angela M Madden 1,* \\ ${ }^{1}$ School of Life and Medical Sciences, University of Herffordshire, Hatfield, Hertfordshire AL10 9AB, UK: ${ }^{2}$ Homerton \\ University Hospitals NHS Foundation Trust, London, UK
}

Submitted 22 August 2017: Final revision received 4 November 2017: Accepted 13 November 2017: First published online 26 December 2017

\begin{abstract}
Objective: To evaluate the nutrient quality of breads and breakfast cereals identified using the wholegrain definition of $\leq 10: 1$ carbohydrate:fibre ratio. Design: Following a cross-sectional study design, nutritional information was systematically gathered from food labels of breads and breakfast cereals that met the $\leq 10: 1$ carbohydrate:fibre criterion. The median nutrient content was compared with the UK Food Standards Agency's nutrient profiling standards and the associations between carbohydrate:fibre ratio and other nutrients were analysed. Subgroup analyses were undertaken for products with and without fruit, nuts and/or seeds. Setting: Products from four major supermarket stores in the UK.

Subjects: Breads ( $n$ 162) and breakfast cereals ( $n$ 266).

Results: Breads which met the $\leq 10: 1$ criterion typically contained medium fat, low saturated fat, low sugar and medium Na. Breakfast cereals typically contained medium fat, low saturated fat, high sugar and low $\mathrm{Na}$. In both groups, as the carbohydrate:fibre ratio decreased, fat content increased (bread: $P=0.029$, $r=-0.171$; breakfast cereal: $P=0.033, r=-0 \cdot 131)$ and, in breakfast cereals, as the ratio increased, sugar content increased $(P<0.0005, r=0.381)$. Breakfast cereals with fruit, nuts and/or seeds contained, per $100 \mathrm{~g}$, more energy $(P=0 \cdot 002)$, fat, saturated fat and sugar (all $P<0.0005$ ), while seeded breads had more energy, fat and saturated fat (all $P<0 \cdot 0005$ ).

Conclusions: Overall, breads and breakfast cereals meeting the $\leq 10: 1$ criterion have good nutritional quality, suggesting that the criterion could be useful in public health and/or food labelling. The utility of applying the $\leq 10: 1$ criterion to products containing fruit, nuts and/or seeds is less clear and requires further research.
\end{abstract}

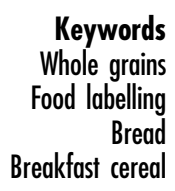

The term 'whole grain' typically refers to the entire edible grain from cereals and related plants ${ }^{(1)}$. Food containing whole grains can include the full grain or be reconstituted so the components of the grain are recombined to a similar proportion to that of the original grain ${ }^{(2)}$. Many studies suggest that foods high in wholegrain ingredients may reduce the risk of chronic diseases such as CVD, type 2 and gestational diabetes and some cancers, including gastrointestinal and breast cancer $^{(2-6)}$. Foods high in whole grains can replace more processed, higherglycaemic-index cereals such as white bread and hence maintain satiation and prolong gastric emptying, which may reduce obesity risk $^{(7)}$. These effects are potentially due to substances in the germ and bran of the grain which work synergistically, including insoluble and soluble fibre, phytosterols and antioxidants such as lignans, polyphenols and flavonoids ${ }^{(8)}$. However, some of the health benefits of whole grains may also be attributed to their role as a proxy for a diet high in other nutrients. For example, Venn and Mann ${ }^{(9)}$ demonstrated uncertainty about whether the impact of unrefined grain intake on reducing diabetesrelated mortality is due to wholegrain foods or other lifestyle factors. Thus, the mechanism by which whole grains are beneficial is still partially unclear. Furthermore, much of the research into whole grains is sponsored by companies that manufacture cereal-based products ${ }^{(2,10,11)}$. While not all of these companies are interested in wholegrain goods, this funding may influence both the focus of cereal-based studies and the findings that are published. In addition, there is little consistency in how authors define wholegrain foods ${ }^{(12)}$. These factors influence conclusions that can be drawn from the literature ${ }^{(12,13)}$. Despite this, there is a general consensus from authoritative organisations that increasing intake of whole 
grains can reduce the risk of many important public health problems ${ }^{(14-16)}$.

Unlike the USA, Australia, Denmark and Canada, the UK does not have a quantified recommendation for intake of whole grains ${ }^{(17)}$. The only recommendation is in The Eatwell Guide, which advises that 'starchy food should make up about a third of the food' eaten in a day and wherever possible, wholegrain options should be cho$\operatorname{sen}^{(14)}$. However, intake of whole grains remains low. Comparison of data from the UK National Diet and Nutrition Survey (NDNS) with the US recommendation of at least three $16 \mathrm{~g}$ servings of whole grains per day shows that $45 \%$ of UK adults ate less than one serving and $18 \%$ consumed no whole grains during the $4 \mathrm{~d}$ collection period ${ }^{(17)}$. With obesity and diabetes increasing ${ }^{(18)}$ and low intakes of whole grains, a public health initiative to substitute foods containing whole grains for those containing significant amounts of refined grains might improve health, save resources and reduce total and CVD-related mortality ${ }^{(19)}$.

However, it is difficult to promote whole grains when there is little standardisation in defining 'wholegrain' foods. In Europe, a food must have $\geq 51 \%$ wholegrain ingredients by wet weight in order to make a health claim ${ }^{(20)}$. However, other nutrients are not specified in relation to this, which means that products making this claim can also have very high levels of sugar or fat. The UK Institute of Grocery Delivery ${ }^{(1)}$ recommends that a food should have at least $8 \mathrm{~g}$ of wholegrain ingredients per serving to be called whole grain. However, as with the USA and Canadian Whole Grain Stamp ${ }^{(21)}$, this recommendation is non-binding. Nonstatutory labelling is predominantly decided by industry representatives ${ }^{(1)}$, which raises the concern that they may focus on commercial rather than health purposes and thus may be potentially misleading ${ }^{(22,23)}$.

In 2015 the UK Scientific Advisory Committee on Nutrition recommended that a standardised definition of "whole grain' and wholegrain foods should be developed as this would facilitate recommended portion sizes for wholegrain foods and support public health messages about the importance of dietary fibre ${ }^{(24)}$. Similarly, the US Dietary Guidelines Technical Advisory Committee has declared 'a call to action' to develop a definition of wholegrain foods that can be internationally implemented ${ }^{(12)}$. This would benefit public health directly by providing consumers with consistent and useful information and indirectly by facilitating research into whole grains ${ }^{(12,25)}$.

Mozaffarian et al. recently compared five different criteria to see which represented the healthiest American wholegrain-based foods ${ }^{(23)}$. They found that foods with a $\leq 10: 1$ carbohydrate:fibre ratio contained the least sugar, $\mathrm{Na}$ and trans-fats. No similar studies have been undertaken outside the USA but this criterion could be considered in other countries.

The $\leq 10: 1$ criterion is based on the recommendation of the American Heart Association ${ }^{(26)}$ as it is approximately the carbohydrate:fibre ratio in whole-wheat flour ${ }^{(23)}$.
This ratio acts as a benchmark to represent the 'balance of whole grain $v$. sugars and refined grains', hence indicating overall carbohydrate quality ${ }^{(23)}$. However, there is no clarification that the fibre content must come from wholegrain ingredients.

The aim of the current cross-sectional study was to evaluate the nutritional quality of breads and breakfast cereals identified using the wholegrain definition of $\leq 10: 1$ carbohydrate:fibre ratio and, therefore, to examine the utility of this criterion by considering the health implications of foods that it defines.

The term 'utility' in this context is used to describe a variety of factors. These include, but are not limited to: how easily the criterion can be used; how healthy the foods that meet it are; whether it can be used to promote healthier choices; and whether it is representative of wholegrain foods. Even though the word 'healthy' is used herein, the authors acknowledge that it is subjective and can have various meanings; this is discussed further in the paper's limitations.

\section{Methods}

\section{Data collection}

The NDNS was used to establish which cereal-based foods are most commonly eaten in the UK. It was determined that breads and breakfast cereals constitute $49 \%$ of UK cereal intake ${ }^{(27)}$. Using these foods to evaluate the use of the criterion was appropriate as they make a major contribution to cereal intake. The remaining $51 \%$ included foods like pasta, rice and sweet items. However, other commonly consumed foods containing whole grains, such as pasta, varied considerably in the form of products available (e.g. being sold dry or fresh), which confounded analysis, so they were not included in the present study. Hence, all the products in the current research were breads or breakfast cereals with a carbohydrate:fibre ratio $\leq 10: 1$. Products with a ratio $>10: 1$ were not studied. Sweet breads (e.g. malt loaf), gluten-free foods and foods marketed for infants and toddlers were excluded due to typically having a different composition.

Nutritional information was obtained online from four major supermarkets to make the results generalisable, as $79 \%$ of the UK population buys food from one or more of these shops ${ }^{(28)}$. Their websites were accessed in order of their market share: Tesco, Asda, Sainsbury's and Morrison's ${ }^{(28)}$. A generic search was made using the terms 'bread' and 'breakfast cereal' and then searches were conducted for more unusual products that the NDNS included such as 'roti' or 'English muffins'. Out of all the breads and breakfast cereals across the supermarkets that met the search terms, a total of 162 breads and 266 breakfast cereals met the criterion and were included in the study. The nutritional information for each product was documented as $\mathrm{g} / 100 \mathrm{~g}$ and $\mathrm{kJ} / 100 \mathrm{~g}$ for comparison. Data included portion size, carbohydrate, fibre, energy, fat, saturated fat, sugar, $\mathrm{Na}$ and ingredients. 
These nutrients were included because they are used to assess a food's nutrient quality ${ }^{(29)}$. Carbohydrate:fibre ratio was calculated.

\section{Data analysis}

Nutritional data were assessed for statistical distribution and, as the data were non-parametric, the median and quartiles of energy and each nutrient were calculated for breads, breakfast cereals and subgroups (listed below). The data for fat, saturated fat, sugar and Na were categorised according to the Food Standards Agency's (FSA) definitions of 'high', 'medium' and 'low' levels of each nutrient per $100 \mathrm{~g}$ as used in UK nutrient profiling ${ }^{(29)}$ (Table 1). Therefore, conclusions were drawn about amounts of nutrients in the foods meeting the criterion. $\mathrm{Na}$ is referred to throughout this paper but was multiplied by 2.5 for conversion to salt (based on $1 \mathrm{~g}$ of salt containing $390 \mathrm{mg} \mathrm{Na}$ ) for comparison with the FSA definitions ${ }^{(30)}$.

The association between the carbohydrate:fibre ratio and the amount of energy, fat, saturated fat, sugar and $\mathrm{Na}$ was examined in breads and breakfast cereals using a Spearman rank correlation.

Subgroup analyses were undertaken using independentsamples median tests as it was observed that some of the products containing fruit, nuts and/or seeds also contained substantial quantities of refined carbohydrate and fewer wholegrain ingredients. The analyses compared breads containing seeds with breads that did not and breakfast cereals containing fruit, nuts and/or seeds with those that did not.

Most of the analyses for breakfast cereals were based on the dry products. However, it was recognised that breakfast cereals are often consumed with milk. As milk contains carbohydrate but negligible fibre ${ }^{(31)}$, a separate analysis was completed in which the carbohydrate in a

Table 1 Food Standard Agency's nutrient profiling categories for high, medium and low amounts of nutrients per $100 \mathrm{~g}$, including salt recalculated as sodium as described in the 'Methods' section ${ }^{(25)}$

\begin{tabular}{|c|c|c|c|c|}
\hline Level & $\begin{array}{c}\text { Fat } \\
(\mathrm{g} / 100 \mathrm{~g})\end{array}$ & $\begin{array}{l}\text { Saturated fat } \\
(\mathrm{g} / 100 \mathrm{~g})\end{array}$ & $\begin{array}{l}\text { Sugar } \\
(\mathrm{g} / 100 \mathrm{~g})\end{array}$ & $\begin{array}{c}\mathrm{Na} \\
(\mathrm{g} / 100 \mathrm{~g})\end{array}$ \\
\hline $\begin{array}{l}\text { Low } \\
\text { Medium } \\
\text { High }\end{array}$ & $\begin{array}{c}<3.0 \\
3 \cdot 0-20.0 \\
>\mathbf{2 0 . 0}\end{array}$ & $\begin{array}{c}\frac{<1.5}{1 \cdot 5-5 \cdot 0} \\
>5 \cdot 0\end{array}$ & $\begin{array}{l}<5.0 \\
5-15 \cdot 0 \\
>15.0\end{array}$ & $\begin{array}{c}\frac{<0.1}{0.1-0.6} \\
>0.6\end{array}$ \\
\hline
\end{tabular}

portion of semi-skimmed milk $(125 \mathrm{ml})^{(31,32)}$ was added to the carbohydrate content of a portion of each breakfast cereal (specified by the manufacturer) to assess how milk affects the carbohydrate:fibre ratio. This was conducted for breakfast cereals containing fruit, nuts and/or seeds and those that did not. The breakfast cereals that exceeded this ratio once milk was added were still included in the research as the carbohydrate:fibre ratio of the dry product was the main focus.

All tests were completed using the statistical software package IBM SPSS Statistics version 22.0 and $P<0.05$ was accepted as statistically significant.

\section{Results}

\section{Comparisons of nutrient content with Food Standards Agency categories}

The nutrient content per $100 \mathrm{~g}$ for 162 breads and 266 breakfast cereals with $\leq 10: 1$ carbohydrate:fibre ratio classified using the FSA ${ }^{(29)}$ high, medium or low categories is presented in Table 2. For bread, the content of saturated fat, sugar and $\mathrm{Na}$ based on median values were consistent enough for generalisations to be made, i.e. that typically they contained low saturated fat, low sugar and medium $\mathrm{Na}$. There was more variation in the fat content, but, based on median values, they contained a medium amount of fat.

For breakfast cereals, the median values indicated medium fat, low saturated fat, high sugar and low Na content. However, for all the nutrients, the values at the 25th, median and/or 75th quartiles corresponded to different FSA categories, indicating that the amounts of nutrients in breakfast cereals are more widely distributed (Table 2).

\section{Relationships between carbohydrate:fibre ratio and energy and nutrient content}

A significant positive correlation was found between the ratio and sugar content for breakfast cereals $(P<0 \cdot 0005$, $r=0.381$ ) indicating that as carbohydrate increases and/or fibre decreases, sugar content increases. A significant negative correlation was found for both breads and breakfast cereals between the ratio and fat (breads: $P=0.029$, $r=-0.171$; breakfast cereals: $P=0.033, \quad r=-0.131)$. This indicates that as carbohydrate decreases and/or fibre increases, fat content increases.

Table 2 Energy and nutrient content per $100 \mathrm{~g}$ of breads and breakfast cereals meeting the $\leq 10: 1$ wholegrain criterion available from four major supermarket stores in the UK, September-November 2015. Values are classified as high, medium or low amounts of nutrients per $100 \mathrm{~g}$, following the Food Standards Agency's nutrient profiling categories ${ }^{(29)}$

\begin{tabular}{|c|c|c|c|c|c|c|c|c|}
\hline Food group & $\begin{array}{c}\text { Carbohydrate } \\
(\mathrm{g} / 100 \mathrm{~g})\end{array}$ & $\begin{array}{l}\text { Fibre } \\
(\mathrm{g} / 100 \mathrm{~g})\end{array}$ & $\begin{array}{l}\text { Carbohydrate: } \\
\text { fibre ratio }\end{array}$ & $\begin{array}{l}\text { Energy } \\
(\mathrm{kJ} / 100 \mathrm{~g})\end{array}$ & $\begin{array}{c}\text { Fat } \\
(\mathrm{g} / 100 \mathrm{~g})\end{array}$ & $\begin{array}{l}\text { Saturated fat } \\
(\mathrm{g} / 100 \mathrm{~g})\end{array}$ & $\begin{array}{l}\text { Sugar } \\
(\mathrm{g} / 100 \mathrm{~g})\end{array}$ & $\begin{array}{c}\mathrm{Na} \\
(\mathrm{g} / 100 \mathrm{~g})\end{array}$ \\
\hline \multicolumn{9}{|l|}{ Breads ( $n$ 162) } \\
\hline 25th quartile & 37.00 & 5.38 & $5 \cdot 29$ & 981.00 & $2 \cdot 40$ & 0.40 & 2.60 & 0.88 \\
\hline Median & $39 \cdot 75$ & $6 \cdot 45$ & $6 \cdot 18$ & $1032 \cdot 50$ & 3.50 & 0.56 & $\overline{3.20}$ & 0.93 \\
\hline 75th quartile & 43.25 & $7 \cdot 10$ & 7.79 & $1159 \cdot 25$ & 6.75 & 1.03 & 4.20 & 1.00 \\
\hline \multicolumn{9}{|c|}{ Breakfast cereals ( $n$ 266) } \\
\hline 25th quartile & 60.08 & $7 \cdot 70$ & 6.75 & 1518.75 & 2.50 & 0.60 & 13.75 & 0.03 \\
\hline Median & $66 \cdot 00$ & 8.50 & 7.57 & 1572.50 & $6 \cdot 20$ & $\overline{1.10}$ & $18 \cdot 40$ & $\overline{0.13}$ \\
\hline 75th quartile & 69.68 & 9.70 & 8.86 & 1682.50 & 9.50 & 1.85 & $22 \cdot 13$ & 0.45 \\
\hline
\end{tabular}


For breads, there were no significant relationships between the ratio and the content of energy, saturated fat, sugar or $\mathrm{Na}$. For breakfast cereals there were no significant relationships between the ratio and energy, saturated fat or Na.

\section{Subgroup analyses}

There were significant differences in the median amounts of nutrients in breads with and without seeds (Table 3). Most importantly, seeded breads had significantly higher energy, fat and saturated fat (all $P<0.0005$ ).

There were also significant differences in the median amounts of nutrients in the breakfast cereals containing fruit, nuts and/or seeds and those without (Table 3). The products containing fruit, nuts and/or seeds had significantly higher energy $(P=0.002)$ and fat, saturated fat and sugar (all $P<0 \cdot 0005$ ).

Without distinguishing between breakfast cereals that did and did not contain fruit, nuts and/or seeds, when the nutritional information of a portion of semi-skimmed milk was added to each breakfast cereal portion, ninety (34\%) exceeded the $\leq 10: 1$ ratio.

\section{Discussion}

The present study aimed to evaluate the nutritional quality of breads and breakfast cereals identified using the wholegrain definition of $\leq 10: 1$ carbohydrate:fibre ratio in order to contribute to understanding of the utility of the criterion in promoting nutritional health. The FSA categories for fat, saturated fat, sugar and salt were chosen for comparison as they are an accepted method of nutrient profiling in the $\mathrm{UK}^{(33)}$ and highly relevant to public health.

\section{Evaluation of the criterion in relation to breads meeting the $\leq 10: 1$ ratio}

The median nutrient content for wholegrain breads identified using the $\leq 10: 1$ ratio suggests they have good nutritional quality, an important indication of the utility of this criterion. The low saturated fat and sugar content supports current public health guidance as limiting saturated fat and sugar intake is recommended to reduce the risk of common long-term conditions in the UK, notably CVD and diabetes ${ }^{(14)}$. This is one reason why wholegrain bread is recommended as a carbohydrate source in guidelines across Europe ${ }^{(14,34)}$. The medium content of fat is not concerning as only $16 \%$ is saturated. The remaining unsaturated fats may promote a healthier blood lipid profile, improving cardiovascular health ${ }^{(35)}$, again suggesting these breads have good nutritional quality. The medium Na content is unsurprising as bread is the largest single contributor of $\mathrm{Na}$ in the $\mathrm{UK}$ diet $^{(36)}$. This is a well-established concern ${ }^{(37)}$. Most breads have a high salt content, not just those meeting the criterion, but this is decreasing in UK bread following a voluntary salt-reduction programme ${ }^{(36)}$.

Studies of wholegrain breads tend to examine health benefits or micronutrient supplementation rather than overall nutritional content as in the present study. In addition, no published studies have examined the $\leq 10: 1$ criterion in UK breads. Results of the present study cannot be compared with the findings of Mozaffarian et al. ${ }^{(23)}$ as their study did not distinguish between the nutritional composition of breads and other products meeting the criterion. However, when compared with wholegrain breads in the UK food tables (i.e. McCance and Widdowson's The Composition of Foods ${ }^{(31)}$ ), the breads that met the criterion were higher in fat, saturated fat, sugar and $\mathrm{Na}$, suggesting that they are, overall, less healthy. Nevertheless, this comparison is limited as the food tables have a smaller sample size and analysis methods may differ. Overall, the nutritional composition of breads meeting the criterion appears to correspond with healthy eating recommendations ${ }^{(14)}$, which indicates the utility of using this criterion.

Regardless of the relatively good nutritional profile and health implications, it is important to consider that, due to

Table 3 Median content of carbohydrate, fibre, carbohydrate: fibre ratio, sugar, sodium, energy, fat and saturated fat for breads with and without seeds, and breakfast cereals with and without fruit, nuts and/or seeds, available from four major supermarket stores in the UK, September-November 2015

\begin{tabular}{|c|c|c|c|c|c|c|c|c|}
\hline Subgroup & $\begin{array}{l}\text { Carbohydrate } \\
(\mathrm{g} / 100 \mathrm{~g})\end{array}$ & $\begin{array}{c}\text { Fibre } \\
(\mathrm{g} / 100 \mathrm{~g})\end{array}$ & $\begin{array}{l}\text { Carbohydrate: } \\
\text { fibre ratio }\end{array}$ & $\begin{array}{l}\text { Energy } \\
(\mathrm{kJ} / 100 \mathrm{~g})\end{array}$ & $\begin{array}{c}\text { Fat } \\
(\mathrm{g} / 100 \mathrm{~g})\end{array}$ & $\begin{array}{l}\text { Saturated fat } \\
(\mathrm{g} / 100 \mathrm{~g})\end{array}$ & $\begin{array}{l}\text { Sugar } \\
(\mathrm{g} / 100 \mathrm{~g})\end{array}$ & $\begin{array}{c}\mathrm{Na} \\
(\mathrm{g} / 100 \mathrm{~g})\end{array}$ \\
\hline \multicolumn{9}{|l|}{ Breads } \\
\hline Bread containing seeds $(n 60)$ & 38.40 & $6 \cdot 70$ & $5 \cdot 76$ & $1165 \cdot 00$ & $7 \cdot 30^{*}$ & 0.90 & $3 \cdot 20$ & 0.37 \\
\hline Bread not containing seeds ( $n$ 102) & 40.50 & $6 \cdot 35$ & $6 \cdot 12$ & 1005.00 & $2 \cdot 60^{*}$ & 0.40 & $3 \cdot 20$ & 0.94 \\
\hline$P$ value $\dagger$ & 0.026 & 0.323 & 0.212 & $<0.0005$ & $<0.0005$ & $<0.0005$ & 0.996 & 0.883 \\
\hline \multicolumn{9}{|l|}{ Breakfast cereals } \\
\hline $\begin{array}{l}\text { Breakfast cereals containing } \\
\text { fruit, nuts and/or seeds ( } n 138)\end{array}$ & 64.45 & $7 \cdot 58$ & $7 \cdot 66$ & $1601 \cdot 50$ & $7 \cdot 55$ & $1 \cdot 20$ & $20 \cdot 05$ & $0 \cdot 10$ \\
\hline $\begin{array}{l}\text { Breakfast cereals without fruit, } \\
\text { nuts and/or seeds }(n 128)\end{array}$ & 68.50 & 8.80 & $7 \cdot 66$ & $1564 \cdot 50$ & $3 \cdot 70$ & 0.85 & $16 \cdot 00$ & 0.24 \\
\hline$P$ value $\ddagger$ & 0.001 & 0.093 & 0.713 & 0.002 & $<0.0005$ & $<0.0005$ & $<0.0005$ & $<0.0005$ \\
\hline
\end{tabular}

*The only difference in Food Standards Agency categories ${ }^{(29)}$ between subgroups is in fat content with seeded and non-seeded breads: seeded breads having medium fat and non-seeded breads having low fat.

$\dagger P$ values obtained by comparing nutrients in breads with seeds and without seeds using the independent-samples median test.

$\ddagger P$ values obtained by comparing nutrients in breakfast cereals containing fruit, nuts and/or seeds with those that did not using the independent-samples median test. 
the inclusion criterion of the $\leq 10: 1$ carbohydrate:fibre ratio, many of the breads contained seeds. While people who eat seeds may have better health (including lower TAG levels and increased insulin sensitivity) ${ }^{(38)}$, seeds contain a different type of fibre from whole grains, which some argue has fewer biological benefits ${ }^{(39)}$. This fibre lowers the ratio without contributing whole grains. In addition, the seeded breads have a different nutritional composition with more energy, fat and saturated fat (Table 3). While eating seeded rather than un-seeded breads is unlikely to have a major impact in isolation on someone's body weight, it may be a less desirable choice for overweight or obese people who are trying to reduce their energy intake. Thirty-seven per cent of the breads examined contained seeds and, based on the food labelling, it is not possible to determine which would meet the $\leq 10: 1$ criterion by virtue of their wholegrain content alone, i.e. if the seeds were excluded. This is a limitation of the criterion as a $\leq 10: 1$ carbohydrate:fibre ratio does not guarantee that a product contains adequate wholegrain ingredients and, as a result, foods meeting the criterion may have a different nutritional profile.

In breads, there was an inverse relationship between the carbohydrate:fibre ratio and fat content. This may relate to the relative mutual displacement of the macronutrients in terms of fat and unrefined carbohydrate or it may be because the seeded breads have more fat and fibre (Table 3 ) due to the contribution from seeds ${ }^{(40)}$. The difference in fibre between breads with and without seeds was not statistically significant, but this may be because of inadequate sample size. In fact, it disagrees with evidence that higher-fibre diets are usually associated with a lower fat intake ${ }^{(23)}$, although this may be because the negative association relates to wholegrain foods alone, not the total diet. Nevertheless, more research is needed to see if this is representative of breads and other wholegrain products. This correlation suggests that, to promote lower-fat wholegrain options, products with a higher ratio should be chosen; however, foods with a higher ratio provide fewer wholegrain benefits due to having less fibre ${ }^{(8)}$.

Even though breads meeting the criterion have a relatively good nutritional profile, the criterion does not account for the negative change to overall nutritional profile due to adding high-fat or high-sugar ingredients such as butter or jam. This is supported by recommendations in international guidelines ${ }^{(41)}$ and recent Public Health England recommendations ${ }^{(14)}$ to avoid adding fats to starchy foods to prevent excess weight gain. Nevertheless, a small serving of butter or jam may not affect health benefits significantly whereas a larger serving could. This is pertinent because bread is normally eaten in a mixed meal which can affect its nutritional properties such as glycaemic index ${ }^{(42)}$, sometimes detracting from potential health benefits of the wholegrain ingredients. Clearly, the utility of the wholegrain criterion is limited by its inability to account for this.

\section{Evaluation of the criterion in relation to breakfast cereals meeting the $\leq 10: 1$ ratio}

There is no other research looking at this criterion in relation to UK breakfast cereals. There is literature that looks at the nutritional composition of wholegrain breakfast cereals, albeit defining whole grains differently, and these are considered here to assist the evaluation. The breakfast cereals meeting the criterion have low amounts of saturated fat and $\mathrm{Na}$ (Table 2) which could contribute beneficially to the diet as the average intake of these nutrients in the UK exceeds recommendations, contributing to poorer heart health ${ }^{(27)}$. This is supported by a systematic review by Williams ${ }^{(43)}$ who found that those who eat wholegrain breakfast cereals have a lower CVD and hypertension risk, partly due to reduced $\mathrm{Na}$ intake. The NDNS ${ }^{(27)}$ has also shown that breakfast cereals, on average, only contribute $1-2 \%$ of total dietary Na. This indicates that the saturated fat and $\mathrm{Na}$ content of these breakfast cereals corresponds with healthy eating recommendations $^{(29)}$. As with breads meeting this criterion, the medium fat content is not of concern as only $18 \%$ is saturated. The remaining unsaturated fats contribute positively to the nutritional profile and may contribute to improved cardiovascular outcomes ${ }^{(44)}$.

Research has shown that, in all age groups, in the UK and internationally, breakfast cereal consumption is associated with reduced overall fat intake ${ }^{(27,45)}$, suggesting that the medium fat content of the breakfast cereals is unlikely to contribute to increased fat intake.

However, some breakfast cereals have a high sugar content which is detrimental to their overall nutritional profile ${ }^{(46)}$. Even though it is well established that some breakfast cereals are high in sugar, there is no evidence that those who eat them have a higher overall sugar intake or are more likely to be overweight ${ }^{(43,47)}$. The present study showed that the higher the carbohydrate:fibre ratio, the higher the sugar content. This may be due to added sugar, listed as an ingredient in $77 \%$ of the breakfast cereals, which contributes to the carbohydrate but not the fibre content. This is supported by Williams ${ }^{(43)}$ who has discussed the presence of sugar in breakfast cereals. However, there are many lower-sugar breakfast cereals as the sugar content of these products varied from 0.3 to $31.4 \mathrm{~g} / 100 \mathrm{~g}$. It could be argued that to make the criterion a better tool, it should only be applied to breakfast cereals that have a $\leq 10: 1$ ratio and a medium or low amount of sugar (i.e. $<15 \mathrm{~g} / 100 \mathrm{~g}$ ). Alternatively, promoting a lower criterion could exclude high-sugar cereals while providing more fibre as suggested by Mozaffarian et al. ${ }^{(23)}$. Furthermore, the UK food tables show that the carbohydrate: fibre ratio of wholemeal flour is typically $6 \cdot 5: 1$ to $7: 1^{(31)}$, which is lower than the $\leq 10: 1$ ratio suggested by the American Heart Association ${ }^{(16)}$. This may be further justification for a lower ratio to be used in order to exclude foods with significant amounts of added sugars. Alternatively, for breakfast cereals, carbohydrate:fibre:sugar 
ratio could be considered to provide a better indication of the overall carbohydrate composition and sugar content.

As with bread meeting this criterion, in these breakfast cereals, as the carbohydrate:fibre ratio decreases, fat content increases. This may be due to nuts and seeds which have high fibre and fat content ${ }^{(40)}$ and was reflected in the study results where breakfast cereals with fruit, nuts and/or seeds contained more energy, fat, saturated fat and sugar (Table 3). Therefore, even though the fruit, nuts and/or seeds can provide benefit through their contribution to micronutrient and phytosterol intake ${ }^{(48,49)}$, the breakfast cereals containing fruit, nuts and/or seeds had a poorer nutritional profile and, considering some may not have met the $\leq 10: 1$ criterion without the addition of fruit, nuts and/or seeds, they may have less wholegrain benefits and hence less bioactive properties. Nevertheless, consuming breakfast cereals with fruit, nuts and/or seeds would still be a good way of increasing the general population's fibre intake, which is an important public health message ${ }^{(27)}$. However, if consumers assumed that all foods with a $\leq 10: 1$ carbohydrate:fibre ratio contain whole grains, they could be misled and choose products high in fruit, nuts and/or seeds but low in actual wholegrain ingredients, especially as some ice creams and fruit juices (both without whole grains) meet this ratio. Another example is a supermarket own-brand porridge pot where the plain porridge did not meet the criterion but the same product with fruit, nuts and/or seeds did. Confusion could be avoided by using clear front-of-pack labelling showing if there is adequate fibre 'from whole grains' to meet the criterion. This would also help consumers identify products that are better for their health. Mozaffarian et al. ${ }^{(23)}$ also supported codifying the $\leq 10: 1$ ratio to use it on front-of-pack labelling.

Furthermore, consideration needs to be given to the type of cereal. As whole wheat typically has a low carbohydrate:fibre ratio (e.g. wholemeal wheat flour, code $11-889=6 \cdot 9)^{(31)}$, this criterion is likely to favour wheat-based foods, along with those with added fibre and rye flour (rye flour, code 11-897 $=5 \cdot 2)^{(31)}$. However, despite oats having a higher carbohydrate:fibre ratio (porridge oats, code $11-788=9 \cdot 1)^{(31)}$ research shows that, due to the presence of $\beta$-glucans and avenanthramides among other bioactive components, there are more consistent results for the health benefits associated with their consumption ${ }^{(50)}$. Clearly identifying the source of the whole grains as well as the carbohydrate:fibre ratio may enable consumers to better understand the overall nutritional value of the food.

Adding other ingredients to breakfast cereals postpurchase alters the carbohydrate:fibre ratio, as with breads. Adding milk can alter the nutritional profile: $34 \%$ of products originally meeting the criterion do not meet it after milk is added. The original premise of the criterion is to capture the balance of whole grains in relation to sugars and refined grains ${ }^{(20)}$ but adding milk detracts from this as lactose in milk increases the ratio. This may look like a drawback of the criterion. However, adding milk helps meet $\mathrm{Ca}$, protein and vitamin $\mathrm{B}$ requirements ${ }^{(51)}$. Lactose also enhances $\mathrm{Ca}$ and $\mathrm{Mg}$ absorption while having a low glycaemic index and carcinogenicity ${ }^{(52)}$. Therefore, even though adding milk to wholegrain breakfast cereals can result in the meal exceeding the $\leq 10: 1$ ratio, it can be argued that the nutrition that milk provides is more important, especially as it does not detract from the benefits of wholegrain ingredients. Adding other ingredients, such as sugar, is different and could detract from the wholegrain ingredients ${ }^{(7)}$. While this is a limitation of the criterion, it may not be worth altering it for this reason. Instead, a separate public health campaign that encourages reducing sugar intake, such as Change4Life's Sugar Smart ${ }^{(53)}$, could mitigate this.

\section{Limitations}

The present study included only breads and breakfast cereals. Other grain-based foods - such as pasta and foods aimed at children aged less than 4 years - could be assessed and evaluating these would contribute to a more comprehensive evaluation of how widely the criterion could be applied. Furthermore, foods from other retailers could have been included since $21 \%$ of the UK population does not shop at the supermarkets surveyed ${ }^{(28)}$ and so there may be products that were not analysed. There are also nutritional components that affect health that are not included on food packaging which could have extended the evaluation; for example, trans-fats or the extent of processing. In addition, the effect of portion size when discussing nutritional content of foods has not been considered. Looking at nutrients per $100 \mathrm{~g}$ enables medians to be calculated and compared with the FSA standards but this does not account for the portion someone might eat. The FSA standards themselves were designed to inform consumers rather than assess food products and have limitations as a tool ${ }^{(54)}$.

In an attempt to discuss the possible health implications of foods meeting this criterion, some inevitable generalisations may have been made regarding the effects of the nutritional composition of wholegrain ingredients or foods on health; these associations are not always straightforward or predictable ${ }^{(55)}$. Another significant limitation is the unavoidable subjectivity when judging health quality. While the authors may consider a medium amount of fat, saturated fat, sugar or Na to represent a relatively healthy food, others may disagree. This indicates the importance of considering the whole diet rather than single components in isolation.

\section{Future recommendations}

As the present study is the first of its kind, more research is needed before steps can be taken towards using this criterion. It would be useful to repeat the research comparing it with similar products with a $>10: 1$ ratio. This would also provide an opportunity to compare the price of foods that meet the criterion with those that do not, to explore the 
cost of foods that meet this criterion and how this could affect sales and intake. The research could also be completed while comparing the nutritional content of the foods with a different nutrient profiling model. This would improve understanding of how useful the criterion is in identifying healthier foods. An investigation into the contribution of fruit, nuts and seeds to products meeting the criterion would also be useful so that decisions can be made about whether products with fruit, nuts and seeds should be included. In addition, more research could be conducted to determine if a different ratio (e.g. $\leq 7: 1$ ) should be used to promote products with less fat or sugar. This could help meet other public health goals. However, a disadvantage of using a criterion other than $\leq 10: 1$ is that it may be more difficult for consumers to work out from food labels if a product meets the wholegrain criterion. It is also important to explore whether consumers would understand how to identify wholegrain foods using this criterion and if not, how it could be adapted. Nevertheless, this $\leq 10: 1$ criterion has potential as a standardised definition for wholegrain foods.

\section{Conclusions}

The present study furthers understanding of the $\leq 10: 1$ carbohydrate:fibre wholegrain criterion and its potential implementation by reporting that foods meeting the $\leq 10: 1$ criterion are relatively healthy when assessed using the FSA nutrient profiling standards as a benchmark; the main exception being the sugar content of breakfast cereals. The utility of the criterion is its potential to offer a standardised approach to the classification of wholegrain breads and breakfast cereals which relates to their nutrient content and thus potentially contributing to efforts to increase wholegrain intake $e^{(20,21,56)}$.

\section{Acknowledgements}

Acknowledgements: The authors would like to thank Dr Alla Mashanova for statistical advice and Dr Helen Crawley for public health feedback. Financial support: This research received no specific grant from any funding agency in the public, commercial or not-for-profit sectors. Conflict of interest: None. Authorship: The research question was proposed by A.M.M.; the study was designed by B.G. supported by A.M.M.; data were collected and analysed by B.G.; the article was written by B.G. and A.M.M. Ethics of human subject participation: This research did not involve human subjects.

\section{References}

1. Institute of Grocery Delivery (2008) UK Whole Grain Guidance. Watford: IGD.

2. Jonnalagadda SS, Harnack L, Liu RH et al. (2011) Putting the whole grain puzzle together: health benefits associated with whole grains - summary of American Society for Nutrition 2010 Satellite Symposium. J Nutr 141, issue 5, 1011S-1022S.

3. Mozaffarian D, Appel LJ \& Van Horn L (2011) Components of a cardioprotective diet: new insights. Circulation 123, 2970-2891.

4. Bao W, Tobias DK, Hu FB et al. (2016) Pre-pregnancy potato consumption and risk of gestational diabetes mellitus: prospective cohort study. BMJ 352, 6898.

5. Farvid MS, Eliassen AH, Cho E et al. (2016) Dietary fiber intake in young adults and breast cancer risk. Pediatrics 137, e20151226.

6. Helnæs A, Kyrø C, Andersen I et al. (2016) Intake of whole grains is associated with lower risk of myocardial infarction: the Danish Diet, Cancer and Health Cohort. Am J Clin Nutr 103, 999-1007.

7. Ludwig DDS (2002) The glycaemic index - physiological mechanisms relating to obesity, diabetes and cardiovascular disease. JAMA 297, 2414-2423.

8. Slavin J (2004) Whole grains and human health. Nutr Res Rev 17, 99-110.

9. Venn BJ \& Mann JI (2004) Cereal grains, legumes and diabetes. Eur J Clin Nutr 58, 1443-1461.

10. Schroeder N, Gallaher DD, Arndt EA et al. (2009) Influence of whole grain barley, whole grain wheat, and refined rice-based foods on short term satiety and energy intake. Appetite 53, 363-369.

11. Giacco R, Clemente G, Cipriano D et al. (2010) Effects of the regular consumption of wholemeal wheat foods on cardiovascular risk factors in healthy people. Nutr Metab Cardiovasc Dis 20, 186-194.

12. Ferruzzi MG, Jonnalagadda SS, Liu S et al. (2014) Developing a standard definition of whole-grain foods for dietary recommendations: a summary report of a multidisciplinary expert roundtable discussion. Adv Nutr 5, 164-176.

13. Fontanarosa PB, Flanagin A \& DeAngelis CD (2005) Reporting conflicts of interest, financial aspects of research and role of sponsors in funded studies. JAMA 294, 110-111.

14. Public Health England (2016) The Eatwell Guide. Helping You Eat a Healthy Balanced Diet. London: Public Health England.

15. World Health Organization (2017) Global Strategy on Diet, Physical Activity and Health. http://www.who.int/dietphysicalactivity/diet/en/ (accessed August 2017).

16. American Heart Association (2016) Whole Grains and Fiber. http://www.heart.org/HEARTORG/HealthyLiving/ HealthyEating/HealthyDietGoals/Whole-Grains-and-Fiber_ UCM_303249_Article.jsp\#.WQ26iNQrLDc (accessed August 2017).

17. Mann KD, Pearce MS, McKevith B et al. (2015) Low whole grain intake in the UK: results from the National Diet and Nutrition Survey rolling programme 2008-11. Br J Nutr $\mathbf{1 1 3}$, 1643-1651.

18. Public Health England (2014) Adult Obesity and Type 2 Diabetes. London: Public Health England.

19. Wu H, Flint AJ, Qi Q et al. (2015) Association between dietary whole grain intake and risk of mortality: two large prospective studies in US men and women. JAMA Intern Med 175, 373-384.

20. European Food Safety Authority (2010) Scientific opinion on the substantiation of health claims related to whole grain. EFSA J 8, 1766-1782.

21. Wholegrains Council (2016) Whole Grain Stamp. http:// www.wholegrainscouncil.org/whole-grain-stamp (accessed August 2017).

22. Gilmore AB, Savell E \& Collin J (2011) Public health, corporations and the new responsibility deal: promoting partnerships with vectors of disease? J Public Health 33, 2-4.

23. Mozaffarian RS, Lee RM, Kennedy MA et al. (2013) Identifying whole grain foods: a comparison of different 
approaches for selecting more healthful whole grain products. Public Health Nutr 16, 2255-2264.

24. Scientific Advisory Committee on Nutrition (2015) Carbohydrates and Health Report. London: Public Health England.

25. Serra-Majem L \& Bautista-Castaño L (2015) Relationship between bread and obesity. Br J Nutr 113, Suppl. 2, S29-S35.

26. Lloyd-Jones DM, Hong Y, Labarthe D et al. (2010) Defining and setting national goals for cardiovascular health promotion and disease reduction: the American Heart Association's Strategic Impact Goal through 2020 and beyond. Circulation 121, 586-613.

27. Public Health England \& Food Standards Agency (2014) National Diet and Nutrition Survey, Results from Years 1, 2, 3 and 4 (combined) of the Rolling Programme (2008/92011/12). London: Public Health England.

28. MINTEL (2014) Food and Drink Retailing - UK. London: Mintel Group Limited.

29. Food Standards Agency (2007) Food. Using Traffic Lights to Make Healthier Choices. London: FSA.

30. Grimes CA, Wright JD, Liu K et al. (2013) Dietary sodium intake is associated with total fluid and sugar-sweetened beverage consumption in US children and adolescents aged 2-18 y: NHANES 2005-2008. Am J Clin Nutr 98, 189-196.

31. Finglas PM, Roe MA, Pinchen HM et al. (2015) McCance and Widdowson's The Composition of Foods: Summary Edition, 7th ed. London: Royal Society of Chemistry.

32. Food Standards Agency (1994) Food Portion Sizes, 3rd ed. London: The Stationery Office.

33. Lobstein T \& Davies S (2009) Defining and labelling 'healthy' and 'unhealthy' food. Public Health Nutr 12, 331-340.

34. van Dooren C \& Kramer G (2012) Food Patterns and Dietary Recommendations in Spain, France and Sweden. Gouda: Blonk Milieu Advies.

35. Vafeiadou K, Weech M, Altowaijri H et al. (2015) Replacement of saturated with unsaturated fats had no impact on vascular function but beneficial effects on lipid biomarkers, E-selectin and blood pressure: results from the randomized, controlled Dietary Intervention and VAScular function (DIVAS) study. Am J Clin Nutr 102, 40-48.

36. Brinsden HC, He FJ, Jenner KH et al. (2013) Surveys of salt content in UK bread: progress made and further reductions possible. BMJ Open 3, e002936.

37. Mhurchu CN, Capelin C, Dunford EK et al. (2011) Sodium content of processed foods in the United Kingdom: analysis of 44,000 foods purchased by 21,000 households. Am J Clin Nutr 93, 594-600

38. Karlsen M, Ellmore GS \& McKeown N (2016) Seeds - health benefits, barriers to incorporation, and strategies for practitioners in supporting consumption among consumers. Nutr Today 51, 50-59.

39. McKee LH \& Latner TA (2000) Underutilized sources of dietary fiber: a review. Plant Foods Hum Nutr 55, 285-304.
40. Anderson JW, Baird P, Davis RH Jr et al. (2009) Health benefits of dietary fiber. Nutr Rev 67, 188-205.

41. Truswell AS (2002) Cereal grains and coronary heart disease. Eur J Clin Nutr 56, 1-14.

42. Dewettinck K, Van Bockstaele F, Kuhne B et al. (2008) Nutritional value of bread: influence of processing, food interaction and consumer perception. J Cereal Sci $\mathbf{4 8}$, 243-257.

43. Williams P (2014) The benefits of breakfast cereal consumption: a systematic review of the evidence base. $A d v$ Nutr 5, issue 5, 636S-673S.

44. Martinez-Gonzalez MA, Salas-Salvado J, Estruch $\mathrm{R}$ et al. (2016) Benefits of the Mediterranean diet: Insights from the PREDIMED study. Prog Cardiovasc Dis 58, 50-60.

45. Ruxton CHS \& Kirk TR (1997) Breakfast: a review of associations with measures of dietary intake, physiology and biochemistry. Br J Nutr 78, 199-213.

46. Lustig RH, Schmidt LA \& Brindis CD (2012) Public health: the toxic truth about sugar. Nature $\mathbf{4 8 2}, 27-29$.

47. McKeown NM, Yoshida M, Shea M et al. (2009) Whole-grain intake and cereal fiber are associated with lower abdominal adiposity in older adults. $J$ Nutr 139, 195-1955.

48. Liu RH (2003) Health benefits of fruit and vegetables are from additive and synergistic combinations of phytochemicals. Am J Clin Nutr 78, 3 Suppl., 517S-520S.

49. Phillips KM, Ruggio DM \& Ashraf-Khorassani M (2005) Phytosterol composition of nuts and seeds commonly consumed in the United States. J Agric Food Chem 53, 9436-9445.

50. Gani A, Wani SM, Masoodi FA et al. (2012) Whole-grain cereal bioactive compounds and their health benefits: a review. J Food Process Technol 3, 146-156.

51. The Dairy Council (2016) Milk Factsheet. London: The Dairy Council.

52. Schaafsma G (2008) Lactose and lactose derivatives as bioactive ingredients in human nutrition. Int Dairy $J \mathbf{1 8}$, 465-468.

53. Change4Life (2015) Let's Get Sugar Smart! http://www.nhs. uk/changeflife-beta/campaigns/sugar-smart/home (accessed August 2017).

54. Scarborough P, Matthews A, Eyles H et al. (2015) Reds are more important than greens: how UK supermarket shoppers use the different information on a traffic light nutrition label in a choice experiment. Int J Behav Nutr Phys Act 12, 151-160.

55. Jacobs DR, Tapsell LC \& Temple NJ (2011) Food synergy: the key to balancing the nutrition research effort. Public Health Rev 33, 507-529.

56. de Munter JSL, Hu FB, Spiegelman D et al. (2007) Whole grain, bran, and germ intake and risk of type 2 diabetes: a prospective cohort study and systematic review. PLOS Med 4, 261. 